\title{
Vigilance by Sentinels in a Group of Baboons (Papio hamadryas hamadryas) in a Zoo Setting
}

\section{Antoni Rebassa ${ }^{1^{*}}$, Colell $\mathrm{M}^{2}$, Munar $\mathrm{E}^{3}$ and Cela $\mathrm{C} \mathrm{J}^{3}$}

${ }^{1}$ Department of Psychology, Centro de Estudios Superiores Alberta Gimenez, Spain

${ }^{2}$ Faculty of Psychology, University of Barcelona, Spain

${ }^{3}$ Department of Psychology, Campus University, Palma de Mallorca

*Corresponding author: Antoni Rebassa, Department of Psychology, Centro de Estudios Superiores Alberta Gimenez, C /Costa de Zaragoza, 16, 07013 Palma de Mallorca, Balearic Islands, Spain, Tel: 971792 818; E-mail: arebassa@cesag.org

Rec date: Jul 11, 2014; Acc date: Aug 12, 2014; Pub date: Aug 16, 2014

Copyright: ( 2014 Rebassa A, et al. This is an open-access article distributed under the terms of the Creative Commons Attribution License, which permits unrestricted use, distribution, and reproduction in any medium, provided the original author and source are credited.

\begin{abstract}
Vigilance by sentinels is an anti-predator strategy by which certain group members keep watch from strategic positions, while the group is involved in other activities. Many anti-predatory behavior patterns observed in natural habitats are also deployed similarly when provoked by human presence. This work is part of a study conducted with a group of hamadryas baboons (Papio $h$. hamadryas) in semi-freedom in a 15-hectare zoo complex with extensive human pressure. The animals make incursions into an adjoining high-risk area to access food resources, making use of vigilance with sentinels and other risk minimization strategies. Results reveal that human presence provokes similar reactions to natural predatory pressure, forcing them to select strategies to minimize the factors involved in risk perception, such as the degree of visibility of the surroundings, minimizing the length of the incursion and behavioral restrictions.
\end{abstract}

Keywords: Sentinels; Vigilance; Papio

\section{Introduction}

Anti-predator vigilance is a visual exploration of the environment with the aim of detecting potential threats and minimizing risk by fleeing [1-3]. In the sentinel system of vigilance, specific group members keep watch from prominent or strategic positions while the other members are involved in other activities, generally feeding [4]. This behavior is seen in many animal species, primarily mammals and birds $[5,6]$ although evidence is almost null among primates or is based on anecdotal or tangential observations $[7,8]$.

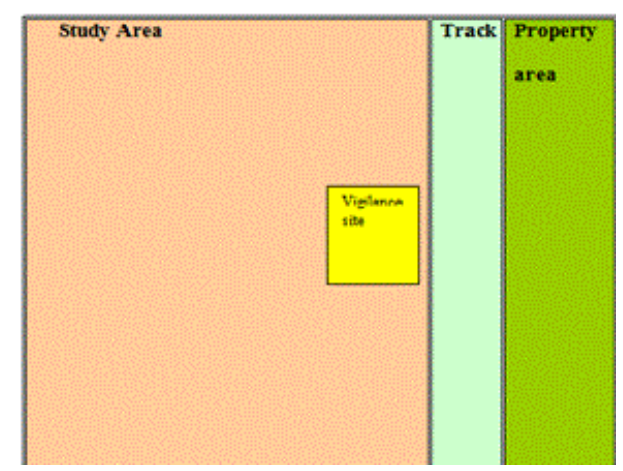

Figure 1: Showing three features

Moreover, a growing number of studies state that many antipredator behaviors that are deployed in natural habitats are also observed in humanized environments [9-14] in which human presence triggers analogue responses in animals to those exhibited with natural predators [9]. Thus, the human influence on animal behavior can be examined in terms of the predatory dynamic, separately considering the factors involved, such as minimizing time investments in risk areas, the optimal feeding time, anti-predator vigilance and/or the decrease/increase of specific behaviors depending on the perceived risk [2]. This study presents preliminary data on the sentinel's behavior in a baboon group in a zoo setting, which has similar traits to those observed in natural conditions.

\section{Material and Methods}

\section{Subjects and study site}

The study was conducted with a group of 31 sacred or Hamadryas baboons (Papio hamadryas hamadryas) in semi-freedom in the Safari Zoo (Majorca, Spain). The group is comprised of three adult males, 13 adult females, and 15 non-adult individuals, distributed in three onemale units (OMUs), prototypical units of social structuring in this species[15] : OMU $1\left(\delta^{\lambda}: 1, \phi+: 8\right)$, OMU 2 ( $\delta^{\lambda}: 1$, , $\left.: 2\right)$, and OMU 3 $(\hat{O}: 1, q: 2)$. The study site is a 15 -hectare pine grove with paths along which visitors drive in their own vehicles. The area of study borders farmlands (property area), which the baboons access for food supplies figs (Ficus carica), almonds (Prunus amygdalus) and carob (Ceratonia siliqua). This incursion has three unusual features (Figure 1) in order to access the property, they have to cross a track used by humans (visitors and caretakers) and get over two physical barriers (a 1.70metre perimeter fence and a 1.50 metre dividing wall), 2) inside the property there is no visibility of the outside (due to visual obstruction from the dividing wall), and 3) the incursion is a critical time, as the protocol to prevent the baboons from climbing the perimeter fence around the grounds is coercion with firearms. 


\section{Procedure}

Data were collected during 473 hours of observation over 16 consecutive months, and are of a larger study on the use of habitat. The adults have been individually recognized and classified in categories by age and gender. The composition of each OMU was determined by using the criterion of sexually exclusive copulation [15]. A behavioral catalogue was created with individual (feeding, rest, movement), social (affiliative, agonistic, submission, sexual) and interspecific (flight and vigilance) categories. To establish the status between OMUs, three data types were triangulated: a) frequency of submissive behavior between male leaders, b) frequency of submissive behavior among females in different subgroups, and c) access frequency to the supplied food. Using scan sampling with continuous sampling during the incursion [16] these data were collected: 1) sentinel subjects 2) vigilance site 3) presence of replacements (swapping of guards and feeders) 4) vigilance type: intensive (vigilance by sustained attention with no alteration with other social and/or individual behaviors) and combined (when they alternate visual scans with other social and/or individual behaviors), 5) date/time of incursions, and 6) length of incursion (minutes).

\section{Results}

A total of 18 incursions into the property were logged with vigilance by sentinels $100 \%$ of the time. The sentinels remained within the study area, while the rest went into the adjoining area, crossing over the perimeter fences. The vigilance site is located at an average distance of 23.5 metres (range: 14-33 m) from the feeding site, from where there is no visibility of the adjoining area, although the rest of the surrounding area is visible. A time restriction was observed with respect to duration, time range and seasonality (Table 1 ). Sentinels were only females in OMU1, the clearly dominant group (Table 2). Vigilance was intensive with an invariable number of sentinels, (2/3 individuals). There were no replacements.

\section{Discussion}

Baboons have vigilance by sentinels system that is deployed in a humanized setting, with the aim of minimizing risks during their incursions. As there is no visibility from inside, the sentinels remain outside, watching out for the approach of humans. This behavior matches that which is observed in natural conditions and entails decision making with respect to the core factors involved in predator risk situations, opting to access food resources with partial entry of the group and using sentinels to compensate for the lack of visibility of the surroundings. Moreover, the time restrictions observed fit the riskassessment hypothesis, according to which the subjects are capable of handling the time to which they are exposed to potential dangers, as a key action to minimize risk [2].

The fact that the sentinels are the females from the dominant subgroup could also fit with Bednekoff's model, which postulates that vigilance by sentinels is based on a cooperative system. This system in turn is due to selfish reasons that depend on the internal state of mind of each sentinel animal in question to ensure their own benefit. Moreover, coordinated vigilance shifts or changing of guards are frequent in this type of vigilance, with little variation in the number of sentinels involved and with a high replacement frequency [17].

\begin{tabular}{|l|l|l|l|l|l|l|l|l|l|l|}
\hline Average duration & \multicolumn{2}{|l|}{ Season } & \multicolumn{2}{l|}{ Time } & \multicolumn{2}{l|}{ Replacements } & \multicolumn{2}{l|}{ Vigilance Type } & \multicolumn{2}{l|}{ No Of Sentinels } \\
\hline & Spring & Summer & Autumn & Winter & $9-12$ & $12-15$ & no & Intensive & 2 & 3 \\
\hline 3.2 & $\begin{array}{l}5.5 \% \\
(\mathrm{n}: 1)\end{array}$ & $50 \%(\mathrm{n}: 9)$ & $\begin{array}{l}38.8 \% \\
(\mathrm{n}: 7)\end{array}$ & $\begin{array}{l}5.5 \% \\
(\mathrm{n}: 1)\end{array}$ & $\begin{array}{l}16.6 \% \\
(\mathrm{n}: 3)\end{array}$ & $\begin{array}{l}82.7 \% \\
(\mathrm{n}: 15)\end{array}$ & $100 \%$ & $100 \%$ & $\begin{array}{l}33.3 \\
\%\end{array}$ & $\begin{array}{l}66.6 \\
\%\end{array}$ \\
\hline
\end{tabular}

Table 1: Characteristics of incursions

\begin{tabular}{|l|l|l|l|}
\hline & OMU 1 & OMU 2 & OMU 3 \\
\hline Submission reception1 (ふ) & 254 & 52 & 26 \\
\hline Submission reception2 (९) & 563 & 8 & 30 \\
\hline Access food3 & $100 \%$ & $0 \%$ & $0 \%$ \\
\hline
\end{tabular}

also attained by minimizing social and individual behaviors that involve a reduction of the visual field [20]. No presence of changing guards is observed, and it is more beneficial for them to feed themselves than to act as sentinels [4]. If we bear in mind the long journey involved between potential replacement guards and those replaced, and the short length of the incursion, the changing of sentinels would hardly be feasible to let the involved baboons have minimum time necessary for ingesting or obtaining supplies. Thus, the

Table 2: Data to determine the relative status of each OMU. (1) Frequency of submission received by the male leader of each OMU, (2) Frequency of submission received by the females of each OMU, (3) Priority access frequency to the food supply (n: 43).

In this case, the number of sentinels remained invariable in all incursions. Some of the variables that can affect the number of sentinels are related to the group size and the conditioning factors in the visual field $[18,19]$. Furthermore, we have ascertained that the critical points from which potential threats could be present are distributed throughout a semicircular geometry around the position occupied by the sentinel and not a single focal point. Thus, two or more individuals can reduce their attention efforts and make sustained attention of these spots possible to increase early detection, which is factors, but also the possibility in terms of the physical layout of the habitat and time restrictions [21,22].

\section{References}

1. Gaynor KM, Cords M (2012) Antipredator and social monitoring functions of vigilance behaviour in blue monkeys. Animal Behaviour 84: 531-537.

2. Lima SL, Dill LM (1990) Behavioural decisions under the risk of predation: A review and prospectus, Can J Zool 68: 619-640.

3. Cowlishaw G (1997) Trade-offs between foraging and predation risk determine habitat use in a desert baboon population. Anim. Behav 53: 667-686. 
4. Bednekoff P (2001) Coordination of safe, selfish sentinels based upon mutual benefits. Annales Zoologici Fennici 38: 5-14.

5. Verdolin J, Slobodchikoff CN (2002) Vigilance and predation risk in Gunnison's prairie dogs. Canadian Journal of Zoology 80: 1197-1203.

6. Newbold T, Collins S, Behnke J (2008). Sentinel behaviour and the watchman's call in the chukar at St Katherine Protectorate, Sinai, Egypt. Egyptian British Biological Society 42-53.

7. Baldellou M, Henzi P (1992) Vigilance, predator detection and the presence of supernumerary males in vervet monkey tropos Anim Behav 43:451-461

8. Horrocks J A, Hunte W (1986) Sentinel behaviour in vervet monkeys: who sees whom first? Anim Behav 34: 1566-1567.

9. Frid A, Dill LM (2002) Human-caused disturbance stimuli as a form of predation risk. Conservation Ecology 6: 11.

10. Sirot E(2010) Should risk allocation strategies facilitate or hinder habituation to nonlethal disturbance in wildlife? Animal Behaviour 80: 737-743

11. Macintosh AJJ, Sicotte P (2009). Vigilance in Ursine Black and White Colobus Monkeys (Colobus vellerosus): An Examination of the Effects of Conspecific Threat and Predation.

12. Beale CM (2007) The behavioural ecology of disturbance responses. International Journal of Comparitve Psychology 20: 111-120.

13. Fernández-Juricic E, Schroeder N (2003) Do variations in scanning behavior affect tolerance to human disturbance? Applied animal behaviour science 84: 219-234
14. Manor R, Saltz D(2005) Effects of human disturbance on use of space and flight distance of mountain gazelles. Journal of wildlife management 69: $1683-1690$

15. Kummer H (1968) Social organisation of Hamadryas baboons. A field study. Basel and Chicago: Karger, University Press. American Journal of Primatology 71: 919-927

16. Martin P, Bateson P(1991) La medición del comportamiento. Ed. Alianza $130-139$

17. McGowan KJ, Woldfenden GE (1989) A sentinel system in the florida scrub-jay. Animal Behaviour 37: 1000-1006.

18. Bednekoff PA (1997) Mutualism among safe, selfish sentinels: a dynamic game. Am Nat 150: 373-392.

19. Fernández-Juricic E, Kowalski V (2011) Where does a flock end from an information perspective? A comparative experiment with live and robotic birds. Behavioral ecology 22: 1304-1311.

20. Fernández-Juricic E, Jokimäki J, McDonald JC, Melado F, Toledano A, et al. (2004) Effects of opportunistic predation on anti-predator behavioural responses in a guild of ground foragers. Oecologia 140: 183-190.

21. Hollén LI, Bell MB, Wade H M, et al. 2011. Ecological conditions influence sentinel decisions. Animal Behaviour 82: 1435-1441.

22. Orpwood JE, Magurran AE, Armstrong JD, and Griffiths SNW(2008) Minnows and the selfish herd: effects of predation risk on shoaling behaviour are dependent on habitat complexity. Animal behavior 76: $143-152$ 